\title{
Heart
}

\subsection{Metabolic Syndrome and Multiple Organ Damage in}

\section{Essential Hypertension}

C. Cuspidi (1), C. Valerio (2), V. Giudici (2), F. Negri (1), C. Sala (3), M. Maisaidi

(4), A. Zanchetti (5), G. Mancia (6)

(1)Istituto Auxologico Italiano e Dipartimento di Medicina Clinica e

Prevenzione, Università di Milano - Bicocca, Milano; (2)Istituto Auxologico

Italiano, Milano; (3)Istituto di Medicina Cardiovascolare, Policlinico IRCCS and

Centro Interuniversitario Fisiologia, Università di Milano, Milano; (4)Istituto di

Medicina Cardiovascolare, Ospedale Maggiore Policlinico Mangiagalli and

IRCCS Regina Elena, Milano; (5)Istituto Auxologico Italiano e Centro

Interuniversitario di Fisiologia Clinica e Ipertensione, Milano; (6)Dipartimento di

Medicina Clinica, Università di Milano - Bicocca, Istituto di Medicina

Cariovascolare, Policlinico di Milano e Centro Interuniversitario di Fisiologia,

Università di Milano, Milano, Italy

Aim. We investigated the prevalence of the metabolic syndrome (MS) in hypertensive patients

categorized according to the number of markers of organ damage (OD) in order to assess the value of a systematic search for cardiac and extra-cardiac OD in the MS setting.

Methods. A total of 3119 untreated and treated essential hypertensive patients included in the Evaluation of Target Organ Damage in Hypertension (ETODH), an observational registry of hypertension-related OD, were considered for this analysis. All patients underwent extensive searching for left ventricular hypertrophy (LVH) or LV concentric remodelling (cardiac OD), carotid plaques and/or intima-media thickening (vascular OD) and microalbuminuria (MA) and/or increased serum creatinine (renal OD). Subjects were classified as: positive for none (group 0), one (group I), two (group II) or three markers (group III) of OD.

Results. MS prevalence rates progressively rose across the groups stratified according to the OD score, reaching a 2.3 -fold increase in group III as compared to their MS counterparts in group 0 . The distribution of subjects with and without the MS across the groups, was 15 vs $29 \%$ (group 0), 32 vs $38 \%$ (group I), 39 vs $26 \%$ (group II) and 14 vs $7 \%$ (group III), respectively. Thus, subjects having 2 or 3 markers of OD were 53\% among those with MS and 33\% ( $\mathrm{p}<0.01)$ among those without it

Conclusions. Our findings indicate a strong association between the MS and OD by showing that a clustering of two or three OD is the prevalent cardiovascular phenotype in MS hypertensive patients referred to a specialist centre and call for a systematic evaluation of cardiac and extra cardiac OD in this setting. 